JOURNAL OF RESEARCH of the National Bureau of Standards - B. Mathematics and Mathematical Physics

Vol. 71B, No. 4, October-December 1967

\title{
Remarks on Cut-Sets
}

\author{
J. W. Grossman* \\ Institute for Applied Technology, National Bureau of Standards, Washington, D.C. 20234
}

(August 14, 1967)

\begin{abstract}
This paper gives a theorem on combinations of segs in a finite, connected, undirected graph. Then the theorem is specialized to combinations of cut sets, giving a theorem first proven by Mayeda. The paper contains an example showing that modifiers added to Mayeda's theorem by Yau, in the Journal of the Franklin Institute, January 1962, yield a false theorem. Finally, the paper discusses the practicability of algorithms developed by Mayeda and Yau and based on Mayeda's theorem.
\end{abstract}

Key Words: Basic cut-sets, cut-sets, graph theory, network flows, mathematics, segs.

This paper, which deals with finite connected undirected graphs, calls attention to an erroneous statement of a theorem of MAYEDA [2] $]^{1}$ made by YAU [4] in The Journal of the Franklin Institute. Counterexamples are given to the theorem as stated. In addition, the theorem is generalized and the proof is given in a more compact form than Mayeda's proof. Finally, the practicability of Mayeda's and Yau's algorithms is discussed.

The fundamental terms of graph theory are used without further explanation in this paper, but several important words are defined to avoid ambiguity:

Definition 1. Let $W$ and $W^{\prime}$ be a partition of the vertices of a graph $G$ into two disjoint nonempty sets. The seg [3], denoted by $\left(W, W^{\prime}\right)$, is the set of all edges with one end point in $W$ and one end point in $W^{\prime}$. The set $W$ (or $W^{\prime}$ ) together with those edges having both end points in $W$ (or $W^{\prime}$ ) will be called a piece. A maximal connected subgraph of a piece or a graph is a component.

Definition 2. A cut set is a seg such that each of the pieces generated by the seg is a component. A cut set may also be defined as a minimal set of edges in a graph such that the removal of this set from the graph divides the graph into two connected subgraphs.

Definition 3. A basic seg (or basic cut set) with respect to two specified vertices $v$ and $w$ is a seg $\left(W, W^{\prime}\right)$ such that $v \epsilon W$ implies $w \epsilon W^{\prime}$. In a connected graph a seg uniquely defines the sets of vertices that determine the two pieces [1; Exercise 1-20]; thus, this definition is unambiguous. A nonbasic seg (or a nonbasic cut set) is a seg such that the two specified vertices $v$ and $w$ are in the same piece $W$.

Definition 4. A fundamental set of cut sets [4] with respect to a spanning tree $T$ of a connected graph $G$ having $v$ vertices is a set of cut sets such that each branch of $T$ is in exactly one of these cut sets, and each of the cut sets includes exactly one branch of $T$. It can be shown that each spanning tree uniquely determines a fundamental set, and that a fundamental set contains $v-1$ cut sets which are linearly independent (i.e., the associated binary vectors are linearly independent modulo 2).

It is well known [1; chap. 5] that any set of $v-1$ linearly independent segs of a graph $G-\operatorname{in}$ particular, any fundamental set of cut sets - will generate all segs (including, of course, all cut sets) by the ring sum operation (i.e., modulo 2 addition of the associated binary vectors).

\footnotetext{
*Consultant. Present address: 2113 South Dinwiddie Street, Arlington, Va. 22206
}

${ }^{1}$ Figures in brackets indicate the literature references at the end of this paper. 
For any subsets $P$ and $Q$ of the vertices of $G$, let $(P, Q)$ denote the set of all edges with one end point in $P$ and the other in $Q$. Referring to figure 1, for any two segs,

$$
S_{1}=\left(A, A^{\prime}\right) \text { and } S_{2}=\left(B, B^{\prime}\right)
$$

we have the representations

$$
\begin{aligned}
S_{1} & =\left(A \cap B, A^{\prime} \cap B\right) \cup\left(A \cap B, A^{\prime} \cap B^{\prime}\right) \cup\left(A \cap B^{\prime}, A^{\prime} \cap B\right) \cup\left(A \cap B^{\prime}, A^{\prime} \cap B^{\prime}\right), \\
S_{2} & =\left(B \cap A, B^{\prime} \cap A\right) \cup\left(B \cap A, B^{\prime} \cap A^{\prime}\right) \cup\left(B \cap A^{\prime}, B^{\prime} \cap A\right) \cup\left(B \cap A^{\prime}, B^{\prime} \cap A^{\prime}\right) \\
& =\left(A \cap B, A \cap B^{\prime}\right) \cup\left(A \cap B, A^{\prime} \cap B^{\prime}\right) \cup\left(A \cap B^{\prime}, A^{\prime} \cap B\right) \cup\left(A^{\prime} \cap B, A^{\prime} \cap B^{\prime}\right)
\end{aligned}
$$

as disjoint unions. Thus,
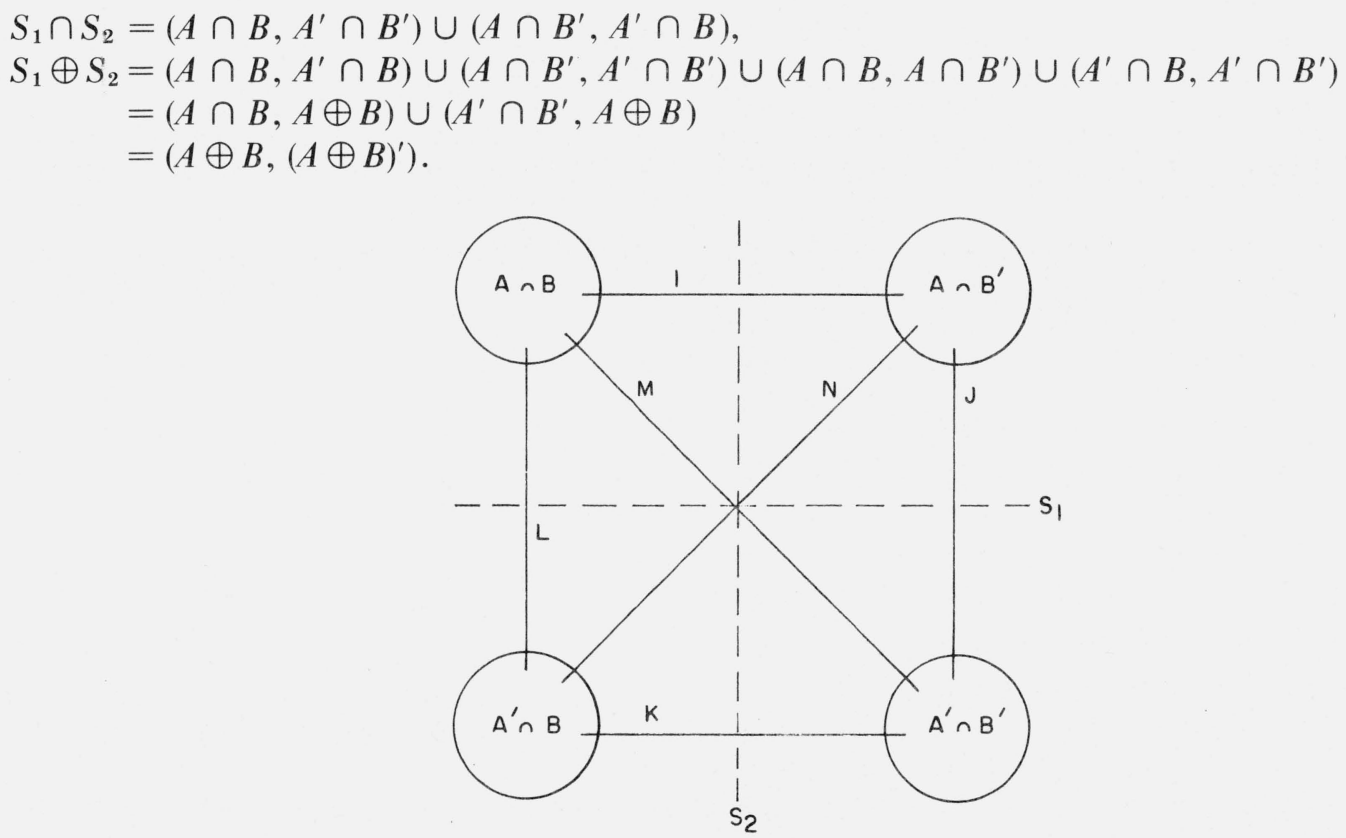

Figure 1. Combination of the two segs $\mathrm{S}_{1}$ and $\mathrm{S}_{2}$.

If $S_{1}$ and $S_{2}$ are distinct segs, so that neither $A=B$ nor $A^{\prime}=B$ holds, then $A \oplus B \neq \emptyset$ and so (since $G$ is connected) $S_{1} \oplus S_{2} \neq \emptyset$. As seen in figure 1, the set of edges of $S_{1} \oplus S_{2}$ is just $I \cup J$ $\cup K \cup L .^{2}$

Using the above notation, the following theorem and corollary are immediate:

THEOREM: Given a graph $\mathrm{G}$, with respect to selected vertices $\mathrm{v}$ and $\mathrm{w}$ :

(a) the ring sum of a basic seg and nonbasic seg is a basic seg;

(b) the ring sum of two basic segs is a nonbasic seg; and

(c) the ring sum of two nonbasic segs is a nonbasic seg.

Proof: Denote the segs, as above, as $S_{1}=\left(A, A^{\prime}\right)$ and $S_{2}=\left(B, B^{\prime}\right)$, so that

$$
S_{1} \oplus S_{2}=\left(A \oplus B,(A \oplus B)^{\prime}\right)
$$

The notation can be chosen so that $v \epsilon A \cap B$; hence $v \epsilon(A \oplus B)^{\prime}$. If $S_{1}$ is basic and $S_{2}$ nonbasic,

${ }^{2}$ In figure 1, it should be noted that the lines $I$, J, etc., represent sets of edges, some of which may be empty. This form of diagram, due to Arthur M. Hobbs of the National Bureau of Standards, was found useful while carrying out the research reported in this paper. 
then $w \in A^{\prime} \cap B \subset A \oplus B$; hence $S_{1} \oplus S_{2}$ is basic. If $S_{1}$ and $S_{2}$ are both basic, then $w \epsilon A^{\prime} \cap B^{\prime}$ $\subset(A \oplus B)^{\prime}$; hence $S_{1} \oplus S_{2}$ is nonbasic. Finally, if $S_{1}$ and $S_{2}$ are nonbasic, then $w \epsilon A \cap B \subset(A \oplus B)^{\prime}$, so again $S_{1} \oplus S_{2}$ is nonbasic.

COROlLARY: (Mayeda) $)^{3}$ Let $\mathrm{G}$ be connected, finite and undirected. Then with respect to a pair of vertices:

(a) the ring sum of a basic cut set and a nonbasic cut set is either a basic cut set or a disjoint union of cut sets, an odd number of which are basic; and

(b) the ring sum of two basic cut sets, or of two nonbasic cut sets, is either a nonbasic cut set or a disjoint union of cut sets, an even number of which are basic.

Proof: Let $S$ and $T$ be two cut sets and let $R=S \oplus T$. Every cut set is a seg; clearly a nonbasic seg cannot be a basic cut set. Thus in both cases, if $R$ is a cut set the theorem implies the corollary immediately. But if $R$ is a seg, it has a disjoint decomposition into cut sets [1]. If one of the cut sets $S$ and $T$ is basic and the other nonbasic, by part (a) of the theorem, $R$ is basic. Suppose some decomposition of $R$ did not contain an odd number of basic cut sets. Then, by parts (b) and (c) of the theorem, $R$ as the ring sum of its decomposition would be nonbasic, a contradiction. Similarly, if $R$ is nonbasic and a decomposition of $R$ contains an odd number of basic cut sets, the ring sum of the decomposition is basic, another contradiction.

Yau [4] stated the above corollary with part (b) phrased as follows: (italics mine)

(ii) the ring sum of two basic cut sets is either a nonbasic cut set or a disjoint union of basic cut sets;

(iii) the ring sum of two nonbasic cut sets is either a nonbasic cut set or a disjoint union of nonbasic edge cut sets.

Mayeda's statement [2] of this corollary does not contain the adjectives italicized above; parts (ii) and (iii) are false with these qualifiers. Figure 2 illustrates a counter-example to YAU's statement of these parts. If the selected vertices are $v=2$ and $w=3$, then cut sets $A$ and $B$ are basic, but $A \oplus B$ can be decomposed only into $C \cup D$, and neither $C$ nor $D$ is basic. Again, if the selected vertices are $v=1$ and $w=4$, the unique decomposition of $A \oplus B$ into $C \cup D$, the disjoint union of basic cut sets, contradicts statement (iii) above.

Although the algorithms presented by MAYEDA and YAU are logically satisfactory, their practicability is another matter. Assuming a Hamiltonian path can be found between $v$ and $w$ in a graph with $n$ vertices, $2^{n-2}$ ring sums must be formed and the results tested for minimality. With $n=50$, not a large graph in modern practice, present-day computers cannot perform the roughly $10^{15}$ ring summations and tests required. This illustrates the serious need in graph theory for improved computational methods necessarily based on more powerful theoretical results.

I thank Alan Goldman and Arthur Hobbs for the valuable advice they gave me during the preparation of this note.

${ }^{3}$ In his statement of this theorem, YAU used the term "branch cut-sets" to distinguish them from cut sets containing vertices. We have dropped the modifier since it is unnecessary here.

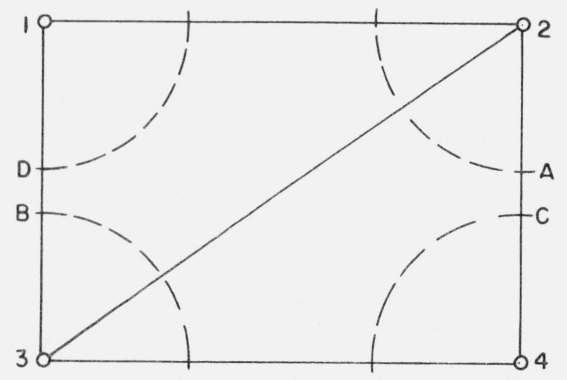

FIGURE 2. Counterexample-Seg A $\oplus \mathrm{B}$ decomposes into cut sets $\mathrm{C}$ and $\mathrm{D}$. 


\section{References}

[1] Robert G. Busacker and Thomas L. Saaty, Finite Graphs and Networks: An Introduction with Applications (McGrawHill Book Co., New York, N.Y., 1965).

[2] Wataru Mayeda, Maximum flow through a communications network, Interim Technical Report No. 13, February 20, 1959, Contract No. DA-11-022-ORD-1983, Circuit Theory Group, Electrical Engineering Research Laboratory, University of Illinois, AD No. 215776.

[3] Myril B. Reed, The seg: a new class of subgraphs, I.R.E. Trans. on Circuit Theory CT-8, No. 1, 17-22 (March 1961).

[4] Sik-Sang Yau, A generalization of the cut-set, Journal of the Franklin Institute 273, No. 1, 31-48, January 1962.

(Paper 71B4-242) 